PROCEEDINGS OF THE

AMERICAN MATHEMATICAL SOCIETY

Volume 137, Number 7, July 2009, Pages 2489-2496

S 0002-9939(09)09809-8

Article electronically published on February 11, 2009

\title{
ON A PRODUCT \\ IN THE CLASSICAL ADAMS SPECTRAL SEQUENCE
}

\author{
XIUGUI LIU AND HAO ZHAO \\ (Communicated by Paul Goerss)
}

\begin{abstract}
In this paper, we prove the nontriviality of the product $h_{0} b_{0} \tilde{\delta}_{s} \in$ $\operatorname{Ext}_{A}^{s+3, t(s)}\left(\mathbb{Z}_{p}, \mathbb{Z}_{p}\right)$ in the classical Adams spectral sequence, where $p \geq 11$, $4 \leq s<p, t(s)=2(p-1)\left[s p^{3}+(s-1) p^{2}+(s-1) p+(s-2)\right]+(s-4)$ and $\tilde{\delta}_{s}$ was obtained by $\mathrm{X}$. Wang and Q. Zheng.
\end{abstract}

\section{INTRODUCTION AND STATEMENT OF RESULTS}

To determine the stable homotopy groups of spheres is one of the most important problems in algebraic topology. So far, several methods have been found to determine the stable homotopy groups of spheres. For example, we have the classical Adams spectral sequence (ASS) (cf. [1]) based on the Eilenberg-MacLane spectrum $K \mathbb{Z}_{p}$, whose $E_{2}$-term is $\operatorname{Ext}_{A}^{s, t}\left(\mathbb{Z}_{p}, \mathbb{Z}_{p}\right)$, and the Adams differential is given by

$$
\tilde{d}_{r}: E_{r}^{s, t} \rightarrow E_{r}^{s+r, t+r-1},
$$

where $A$ denotes the mod $p$ Steenrod algebra. There are three problems in using the ASS: calculation of the $E_{2}$-term $\operatorname{Ext}_{A}^{*, *}\left(\mathbb{Z}_{p}, \mathbb{Z}_{p}\right)$, computation of the differentials and determination of the nontrivial extensions from $E_{\infty}$ to the stable homotopy groups of spheres. So, for computing the stable homotopy groups of spheres with the classical ASS, we must compute the $E_{2}$-term of the ASS, $\operatorname{Ext}_{A}^{*, *}\left(\mathbb{Z}_{p}, \mathbb{Z}_{p}\right)$.

Throughout this paper, $p$ denotes an odd prime and $q=2(p-1)$.

The known results on $\operatorname{Ext}_{A}^{*, *}\left(\mathbb{Z}_{p}, \mathbb{Z}_{p}\right)$ are as follows. $\operatorname{Ext}_{A}^{0, *}\left(\mathbb{Z}_{p}, \mathbb{Z}_{p}\right)=\mathbb{Z}_{p}$ by its definition. From 6$], \operatorname{Ext}_{A}^{1, *}\left(\mathbb{Z}_{p}, \mathbb{Z}_{p}\right)$ has $\mathbb{Z}_{p}$-basis consisting of $a_{0} \in \operatorname{Ext}_{A}^{1,1}\left(\mathbb{Z}_{p}, \mathbb{Z}_{p}\right)$, $h_{i} \in \operatorname{Ext}_{A}^{1, p^{i} q}\left(\mathbb{Z}_{p}, \mathbb{Z}_{p}\right)$ for all $i \geq 0$ and $\operatorname{Ext}_{A}^{2, *}\left(\mathbb{Z}_{p}, \mathbb{Z}_{p}\right)$ has $\mathbb{Z}_{p}$-basis consisting of $\alpha_{2}$, $a_{0}^{2}, a_{0} h_{i}(i>0), g_{i}(i \geq 0), k_{i}(i \geq 0), b_{i}(i \geq 0)$, and $h_{i} h_{j}(j \geq i+2, i \geq 0)$ whose internal degrees are $2 q+1,2, p^{i} q+1, p^{i+1} q+2 p^{i} q, 2 p^{i+1} q+p^{i} q, p^{i+1} q$ and $p^{i} q+p^{j} q$ respectively. In 1980, Aikawa [2] determined $\operatorname{Ext}_{A}^{3, *}\left(\mathbb{Z}_{p}, \mathbb{Z}_{p}\right)$ by $\lambda$-algebra.

In 1998, X. Wang and Q. Zheng [8] proved the following theorem.

Received by the editors September 3, 2008, and, in revised form, October 16, 2008.

2000 Mathematics Subject Classification. Primary 55Q45.

Key words and phrases. Stable homotopy groups of spheres, Adams spectral sequence, May spectral sequence.

The first author was supported in part by the National Natural Science Foundation of China (Nos. 10501045, 10771105) and the Fund of the Personnel Division of Nankai University. 
Theorem 1.1 (Wang and Zheng). For $p \geq 11$ and $4 \leq s<p$, there exists the fourth Greek letter family element $\tilde{\delta}_{s} \neq 0 \in \operatorname{Ext}_{A}^{s, t_{1}(s)}\left(\mathbb{Z}_{p}, \mathbb{Z}_{p}\right)$, where $t_{1}(s)=$ $2(p-1)\left[s p^{3}+(s-1) p^{2}+(s-2) p+(s-3)\right]+(s-4)$.

Note that we write $\tilde{\delta}_{s}$ for $\tilde{\alpha}_{s}^{(4)}$, which is described in [8].

In this note, our main result can be stated as follows.

Theorem 1.2. For $p \geq 11$ and $4 \leq s<p$, the product $h_{0} b_{0} \tilde{\delta}_{s} \neq 0$ in the classical Adams spectral sequence.

The method of proof is by explicit combinatorial analysis of the May spectral sequence (MSS). The method was first given in 4] by the first author, who obtained many other results using it. See [4, 5 .

The paper is arranged as follows: after recalling some knowledge on the MSS in Section 2, we give the proof of Theorem 1.2 in Section 3.

\section{The May spectral Sequence}

As we know, the most successful method to compute $\operatorname{Ext}_{A}^{*, *}\left(\mathbb{Z}_{p}, \mathbb{Z}_{p}\right)$ is the MSS. From [7, there is a May spectral sequence (MSS) $\left\{E_{r}^{s, t, *}, d_{r}\right\}$ which converges to $\operatorname{Ext}_{A}^{s, t}\left(\mathbb{Z}_{p}, \mathbb{Z}_{p}\right)$ with $E_{1}$-term

$$
E_{1}^{*, *, *}=E\left(h_{m, i} \mid m>0, i \geq 0\right) \otimes P\left(b_{m, i} \mid m>0, i \geq 0\right) \otimes P\left(a_{n} \mid n \geq 0\right),
$$

where $E()$ is the exterior algebra, $P()$ is the polynomial algebra, and

$$
h_{m, i} \in E_{1}^{1,2\left(p^{m}-1\right) p^{i}, 2 m-1}, \quad b_{m, i} \in E_{1}^{2,2\left(p^{m}-1\right) p^{i+1}, p(2 m-1)}, \quad a_{n} \in E_{1}^{1,2 p^{n}-1,2 n+1} .
$$

One has

$$
d_{r}: E_{r}^{s, t, u} \rightarrow E_{r}^{s+1, t, u-r}
$$

and if $x \in E_{r}^{s, t, *}$ and $y \in E_{r}^{s^{\prime}, t^{\prime}, *}$, then

$$
d_{r}(x \cdot y)=d_{r}(x) \cdot y+(-1)^{s} x \cdot d_{r}(y) .
$$

In particular, the first May differential $d_{1}$ is given by

$$
d_{1}\left(h_{i, j}\right)=\sum_{0<k<i} h_{i-k, k+j} h_{k, j}, d_{1}\left(a_{i}\right)=\sum_{0 \leq k<i} h_{i-k, k} a_{k}, d_{1}\left(b_{i, j}\right)=0 .
$$

There also exists a graded commutativity in the MSS: $x \cdot y=(-1)^{s s^{\prime}+t t^{\prime}} y \cdot x$ for $x, y=h_{m, i}, b_{m, i}$ or $a_{n}$.

For each element $x \in E_{1}^{s, t, u}$, we define $\operatorname{dim} x=s$, deg $x=t, M(x)=u$. Then we have that

$$
\left\{\begin{array}{l}
\operatorname{dim} h_{i, j}=\operatorname{dim} a_{i}=1 \\
\operatorname{dim} b_{i, j}=2, \operatorname{deg} a_{0}=1 \\
\operatorname{deg} h_{i, j}=q\left(p^{i+j-1}+\cdots+p^{j}\right) \\
\operatorname{deg} b_{i, j}=q\left(p^{i+j}+\cdots+p^{j+1}\right) \\
\operatorname{deg} a_{i}=q\left(p^{i-1}+\cdots+1\right)+1 \\
M\left(h_{i, j}\right)=M\left(a_{i-1}\right)=2 i-1, \\
M\left(b_{i, j}\right)=(2 i-1) p
\end{array}\right.
$$

where $i \geq 1, j \geq 0$. 
Note that by the knowledge on the $p$-adic expression in number theory, for each integer $t \geq 0$, it can be expressed uniquely as

$$
t=q\left(c_{n} p^{n}+c_{n-1} p^{n-1}+\cdots+c_{1} p+c_{0}\right)+e
$$

where $0 \leq c_{i}<p(0 \leq i<n), p>c_{n}>0,0 \leq e<q$.

\section{Proof of Theorem 1.2}

Before showing Theorem 1.2, we first prove some important lemmas which will be used in the proof of Theorem 1.2.

Lemma 3.1. Let $p \geq 11,4 \leq s<p$. Then the fourth Greek letter family element $\tilde{\delta}_{s} \in \operatorname{Ext}_{A}^{s, t_{1}(s)}\left(\mathbb{Z}_{p}, \mathbb{Z}_{p}\right)$ is represented by

$$
a_{4}^{s-4} h_{4,0} h_{3,1} h_{2,2} h_{1,3} \in E_{1}^{s, t_{1}(s), *}
$$

in the MSS, where $t_{1}(s)=q\left[s p^{3}+(s-1) p^{2}+(s-2) p+(s-3)\right]+(s-4)$.

Proof. By Theorem 1.1, it suffices to prove that the May $E_{1}$-term

$$
E_{1}^{s, t_{1}(s), *}=\mathbb{Z}_{p}\left\{a_{4}^{s-4} h_{4,0} h_{3,1} h_{2,2} h_{1,3}\right\} .
$$

This can be shown by an argument similar to that used in Lemma 3.2 below. The proof is left to the reader.

Lemma 3.2. Let $p \geq 11,4 \leq s<p$. Then the May $E_{1}$-term

$$
E_{1}^{s+2, t(s), *}=\mathbb{Z}_{p}\{\mathbf{G} 1, \mathbf{G} 2, \cdots, \mathbf{G} 6\},
$$

where $t(s)=q\left[s p^{3}+(s-1) p^{2}+(s-1) p+(s-2)\right]+(s-4)$, and

$$
\begin{array}{lll}
\text { G1 }=a_{4}^{s-4} h_{4,0} h_{3,1} h_{2,2} h_{1,3} h_{1,1} h_{1,0}, & \text { G2 }=a_{4}^{s-4} b_{3,0} h_{4,0} h_{3,1} h_{1,3} h_{1,0}, \\
\text { G3 }=a_{4}^{s-4} b_{3,0} h_{4,0} h_{2,2} h_{1,3} h_{2,0}, & \text { G4 }=a_{4}^{s-4} b_{1,2} h_{4,0} h_{3,1} h_{2,2} h_{2,0}, \\
\text { G5 }=a_{4}^{s-4} b_{1,2} h_{4,0} h_{3,1} h_{1,3} h_{3,0}, & \text { G6 }=a_{4}^{s-4} b_{2,1} h_{4,0} h_{3,1} h_{1,3} h_{2,0} .
\end{array}
$$

Proof. Consider $h=x_{1} x_{2} \cdots x_{m} \in E_{1}^{s+2, t(s), *}$ in the MSS, where $x_{i}$ is one of $a_{k}$, $h_{r, j}$ or $b_{u, z}, 0 \leq k \leq 4,0 \leq r+j \leq 4,0 \leq u+z \leq 3, r>0, j \geq 0, u>0, z \geq 0$. By (2.5) we can assume that $\operatorname{deg} x_{i}=q\left(c_{i, 3} p^{3}+c_{i, 2} p^{2}+c_{i, 1} p+c_{i, 0}\right)+e_{i}$, where $c_{i, j}=0$ or $1, e_{i}=1$ if $x_{i}=a_{k_{i}}$, or $e_{i}=0$. It follows that

$$
\operatorname{dim} h=\sum_{i=1}^{m} \operatorname{dim} x_{i}=s+2
$$

and

$$
\begin{aligned}
\operatorname{deg} h & =\sum_{i=1}^{m} \operatorname{deg} x_{i} \\
& =q\left[\left(\sum_{i=1}^{m} c_{i, 3}\right) p^{3}+\left(\sum_{i=1}^{m} c_{i, 2}\right) p^{2}+\left(\sum_{i=1}^{m} c_{i, 1}\right) p+\left(\sum_{i=1}^{m} c_{i, 0}\right)\right]+\left(\sum_{i=1}^{m} e_{i}\right) \\
& =q\left[s p^{3}+(s-1) p^{2}+(s-1) p+(s-2)\right]+(s-4) .
\end{aligned}
$$

Note that $\operatorname{dim} h_{i, j}=\operatorname{dim} a_{i}=1, \operatorname{dim} b_{i, j}=2$ and $4 \leq s<p$. From $\operatorname{dim} h=$ $\sum_{i=1}^{m} \operatorname{dim} x_{i}=s+2$ we must have $m \leq s+2 \leq p+1$. Using $0 \leq s, s-1, s-2, s-4<p$ 
and the knowledge on the $p$-adic expression in number theory, we have that

$$
\left\{\begin{array}{l}
\sum_{i=1}^{m} e_{i}=s-4 \\
\sum_{i=1}^{m} c_{i, 0}=s-2 \\
\sum_{i=1}^{m} c_{i, 1}=s-1 \\
\sum_{i=1}^{m} c_{i, 2}=s-1 \\
\sum_{i=1}^{m} c_{i, 3}=s
\end{array}\right.
$$

By $c_{i, 3}=0$ or 1 , one has $m \geq s$ from $\sum_{i=1}^{m} c_{i, 3}=s$. Note that $m \leq s+2$. Thus $m$ may equal $s, s+1$ or $s+2$. Since $\sum_{i=1}^{m} e_{i}=s-4, \operatorname{deg} h_{i, j} \equiv 0(\bmod q)$ $(i>0, j \geq 0), \operatorname{deg} a_{i} \equiv 1(\bmod q)(i \geq 0)$ and $\operatorname{deg} b_{i, j} \equiv 0(\bmod q)(i>0, j \geq 0)$, then by the graded commutativity of $E_{1}^{*, *, *}$ and degree reasons, we can assume that $h=a_{0}^{x} a_{1}^{y} a_{2}^{z} a_{3}^{k} a_{4}^{l} h^{\prime}$ with $h^{\prime}=x_{s-3} x_{s-2} \cdots x_{m}$, where $0 \leq x, y, z, k, l \leq s-4$, $x+y+z+k+l=s-4$. Consequently, we have $h^{\prime}=x_{s-3} x_{s-2} \cdots x_{m} \in E_{1}^{6, t_{2}(s), *}$, where $t_{2}(s)=q\left[(s-l) p^{3}+(s-1-l-k) p^{2}+(s-1-l-k-z) p+(s-2-l-k-z-y)\right]$. From (3.1) we have

$$
\left\{\begin{array}{l}
\sum_{i=s-3}^{m} e_{i}=0 \\
\sum_{i=s-3}^{m} c_{i, 0}=s-2-y-z-k-l \\
\sum_{i=s-3}^{m} c_{i, 1}=s-1-z-k-l \\
\sum_{i=s-3}^{m} c_{i, 2}=s-1-k-l \\
\sum_{i=s-3}^{m} c_{i, 3}=s-l
\end{array}\right.
$$

Case $1(m=s)$. From $\sum_{i=s-3}^{s} c_{i, 3}=s-l$ in (3.1), we have that $l=s-$ $\sum_{i=s-3}^{s} c_{i, 3} \geq s-4$. Note that $0 \leq l \leq s-4$. Thus $l=s-4$, and $x=$ $y=z=k=0$. By $(3.2), h^{\prime}=x_{s-3} x_{s-2} x_{s-1} x_{s} \in E_{1}^{6, q\left(4 p^{3}+3 p^{2}+3 p+2\right), *}$. However, $E_{1}^{6, q\left(4 p^{3}+3 p^{2}+3 p+2\right), *}$ is the $\mathbb{Z}_{p}$-module generated by the following six elements: $h_{4,0} h_{3,1} h_{2,2} h_{1,3} h_{1,1} h_{1,0} ; b_{3,0} h_{4,0} h_{3,1} h_{1,3} h_{1,0} ; b_{3,0} h_{4,0} h_{2,2} h_{1,3} h_{2,0} ; b_{1,2} h_{4,0} h_{3,1} h_{2,2} h_{2,0}$; $b_{1,2} h_{4,0} h_{3,1} h_{1,3} h_{3,0}$; and $b_{2,1} h_{4,0} h_{3,1} h_{1,3} h_{2,0}$, denoted by g1, g2, $\cdots$, g6 respectively. Consequently, in this case it is impossible for $h^{\prime}$ to exist. Then $h$ doesn't exist either.

Case $2(m=s+1)$. From $\sum_{i=s-3}^{s+1} c_{i, 3}=s-l$ in (3.2), one has $l=s-\sum_{i=s-3}^{s+1} c_{i, 3} \geq$ $s-5$. Thus there are five possibilities satisfying $0 \leq x, y, z, k, l \leq s-4$ and $x+y+z+k+l=s-4$. We list all the possibilities in Table 1, consequently, in this case up to sign $h=a_{4}^{s-4} \mathbf{g} 2, a_{4}^{s-4} \mathbf{g} 3, \cdots, a_{4}^{s-4} \mathbf{g} 6$, denoted by $\mathbf{G} 2, \mathbf{G} 3, \cdots, \mathbf{G} 6$ respectively.

Case $3(m=s+2)$. As in Case 2 , one has $l \geq s-6$ from $\sum_{i=s-3}^{s+2} c_{i, 3}=s-l$ in (3.2). Note that $x+y+z+k+l=s-4$. We list all the possibilities in Table 2, thus in this case up to sign $h=a_{4}^{s-4} \mathbf{g} 1$, denoted by $\mathbf{G} 1$. 
TABLE 1

\begin{tabular}{|l|c|c|c|c|c|c|c|}
\hline The possibility & $l$ & $k$ & $z$ & $y$ & $x$ & $E_{1}^{6, t_{2}(s), *}$ & $\begin{array}{c}\text { The existence of } \\
h^{\prime}=x_{s-3} \cdots x_{s+1}\end{array}$ \\
\hline The 1st & $s-5$ & 1 & 0 & 0 & 0 & $E_{1}^{6, q\left(5 p^{3}+3 p^{2}+3 p+2\right), *}=0$ & Nonexistence \\
\hline The 2nd & $s-5$ & 0 & 1 & 0 & 0 & $E_{1}^{6, q\left(5 p^{3}+4 p^{2}+3 p+2\right), *}=0$ & Nonexistence \\
\hline The 3rd & $s-5$ & 0 & 0 & 1 & 0 & $E_{1}^{6, q\left(5 p^{3}+4 p^{2}+4 p+2\right), *}=0$ & Nonexistence \\
\hline The 4th & $s-5$ & 0 & 0 & 0 & 1 & $E_{1}^{6, q\left(5 p^{3}+4 p^{2}+4 p+3\right), *}=0$ & Nonexistence \\
\hline The 5th & $s-4$ & 0 & 0 & 0 & 0 & $\begin{array}{c}E_{1}^{6, q\left(4 p^{3}+3 p^{2}+3 p+2\right), *} \\
=\mathbb{Z}_{p}\{\mathbf{g} 1, \mathbf{g} 2, \cdots, \mathbf{g} 6\}\end{array}$ & $\begin{array}{c}h^{\prime}=\mathbf{g} 2, \mathbf{g} 3, \mathbf{g} 4, \mathbf{g} 5, \\
\mathbf{g} 6 \text { up to sign }\end{array}$ \\
\hline
\end{tabular}

TABLE 2

\begin{tabular}{|c|c|c|c|c|c|c|c|}
\hline The possibility & $l$ & $k$ & $z$ & $y$ & $x$ & $E_{1}^{6, t_{2}(s), *}$ & $\begin{array}{l}\text { The existence of } \\
h^{\prime}=x_{s-3} \cdots x_{s+2} \\
\end{array}$ \\
\hline The 1st & $s-6$ & 2 & 0 & 0 & 0 & $E_{1}^{6, q\left(6 p^{3}+3 p^{2}+3 p+2\right), *}=0$ & Nonexistence \\
\hline The 2nd & $s-6$ & 0 & 2 & 0 & 0 & $E_{1}^{6, q\left(6 p^{3}+5 p^{2}+3 p+2\right), *}=0$ & Nonexistence \\
\hline The 3rd & $s-6$ & 0 & 0 & 2 & 0 & $E_{1}^{6, q\left(6 p^{3}+5 p^{2}+5 p+2\right), *}=0$ & Nonexistence \\
\hline The 4 th & $s-6$ & 0 & 0 & 0 & 2 & $E_{1}^{6, q\left(6 p^{3}+5 p^{2}+5 p+4\right), *}=0$ & Nonexistence \\
\hline The 5th & $s-6$ & 1 & 1 & 0 & 0 & $E_{1}^{6, q\left(6 p^{3}+4 p^{2}+3 p+2\right), *}=0$ & Nonexistence \\
\hline The 6th & $s-6$ & 1 & 0 & 1 & 0 & $E_{1}^{6, q\left(6 p^{3}+4 p^{2}+4 p+2\right), *}=0$ & Nonexistence \\
\hline The 7th & $s-6$ & 1 & 0 & 0 & 1 & $E_{1}^{6, q\left(6 p^{3}+4 p^{2}+4 p+3\right), *}=0$ & Nonexistence \\
\hline The 8th & $s-6$ & 0 & 1 & 1 & 0 & $E_{1}^{6, q\left(6 p^{3}+5 p^{2}+4 p+2\right), *}=0$ & Nonexistence \\
\hline The 9th & $s-6$ & 0 & 1 & 0 & 1 & $E_{1}^{6, q\left(6 p^{3}+5 p^{2}+4 p+3\right), *}=0$ & Nonexistence \\
\hline The 10th & $s-6$ & 0 & 0 & 1 & 1 & $E_{1}^{6, q\left(6 p^{3}+5 p^{2}+5 p+3\right), *}=0$ & Nonexistence \\
\hline The 11th & $s-5$ & 1 & 0 & 0 & 0 & $E_{1}^{6, q\left(5 p^{3}+3 p^{2}+3 p+2\right), *}=0$ & Nonexistence \\
\hline The 12 th & $s-5$ & 0 & 1 & 0 & 0 & $E_{1}^{6, q\left(5 p^{3}+4 p^{2}+3 p+2\right), *}=0$ & Nonexistence \\
\hline The 13 th & $s-5$ & 0 & 0 & 1 & 0 & $E_{1}^{6, q\left(5 p^{3}+4 p^{2}+4 p+2\right), *}=0$ & Nonexistence \\
\hline The 14th & $s-5$ & 0 & 0 & 0 & 1 & $E_{1}^{6, q\left(5 p^{3}+4 p^{2}+4 p+3\right), *}=0$ & Nonexistence \\
\hline The 15th & $s-4$ & 0 & 0 & 0 & 0 & $\begin{aligned} & E_{1}^{6, q\left(4 p^{3}+3 p^{2}+3 p+2\right), *} \\
= & \mathbb{Z}_{p}\{\mathbf{g} 1, \mathbf{g} 2, \cdots, \mathbf{g} 6\}\end{aligned}$ & $h^{\prime}=\mathrm{g} 1$ up to sign \\
\hline
\end{tabular}

Combining Cases 1-3, we obtain that $E_{1}^{s+2, t(s), *}=\mathbb{Z}_{p}\{\mathbf{G} 1, \mathbf{G} 2, \cdots, \mathbf{G} 6\}$. This completes the proof of Lemma 3.2.

Lemma 3.3. (1) The product $h_{0} b_{0} \tilde{\delta}_{s} \in \operatorname{Ext}_{A}^{s+3, t(s)}\left(\mathbb{Z}_{p}, \mathbb{Z}_{p}\right)$ is represented by $h_{1,0} b_{1,0} a_{4}^{s-4} h_{4,0} h_{3,1} h_{2,2} h_{1,3} \in E_{1}^{s+3, t(s), *}$ in the $M S S$, where $t(s)=q\left[s p^{3}+\right.$ $\left.(s-1) p^{2}+(s-1) p+(s-2)\right]+(s-4)$.

(2) For the six generators of $E_{1}^{s+2, t(s), *}$, we have that $M(\mathbf{G} 1)=9 s-18$, $M(\mathbf{G} 2)=M(\mathbf{G} 3)=5 p+9 s-22, M(\mathbf{G} 4)=M(\mathbf{G} 5)=p+9 s-18$, and $M(\mathbf{G} 6)=3 p+9 s-20$. Moreover, for $h_{1,0} b_{1,0} a_{4}^{s-4} h_{4,0} h_{3,1} h_{2,2} h_{1,3}$ we have that $M\left(h_{1,0} b_{1,0} a_{4}^{s-4} h_{4,0} h_{3,1} h_{2,2} h_{1,3}\right)=p+9 s-19$.

Proof. (1) Since it is known that $h_{1, i}, b_{1, i}$ and $a_{4}^{s-4} h_{4,0} h_{3,1} h_{2,2} h_{1,3} \in E_{1}^{*, * * *}$ are all permanent cycles in the MSS and converge nontrivially to $h_{i}, b_{i}, \tilde{\delta}_{s} \in \operatorname{Ext}_{A}^{* * *}\left(\mathbb{Z}_{p}, \mathbb{Z}_{p}\right)$ for $4 \leq s<p$ and $i \geq 0$ respectively (cf. Lemma 3.1), then we have that 
$h_{1,0} b_{1,0} a_{4}^{s-4} h_{4,0} h_{3,1} h_{2,2} h_{1,3} \in E_{1}^{s+3, t(s), p+9 s-19}$ is a permanent cycle in the MSS and converges to $h_{0} b_{0} \tilde{\delta}_{s} \in \operatorname{Ext}_{A}^{s+3, t(s)}\left(\mathbb{Z}_{p}, \mathbb{Z}_{p}\right)$.

(2) From (2.5), the result follows by direct calculation.

Now we give the proof of Theorem 1.2.

Proof of Theorem 1.2. From Lemma $3.3(1), h_{0} b_{0} \tilde{\delta}_{s} \in \operatorname{Ext}_{A}^{s+3, t(s)}\left(\mathbb{Z}_{p}, \mathbb{Z}_{p}\right)$ is represented by $h_{1,0} b_{1,0} a_{4}^{s-4} h_{4,0} h_{3,1} h_{2,2} h_{1,3} \in E_{1}^{s+3, t(s), p+9 s-19}$ in the MSS. Now we will show that nothing hits the permanent cycle $h_{1,0} b_{1,0} a_{4}^{s-4} h_{4,0} h_{3,1} h_{2,2} h_{1,3}$ under the May differential $d_{r}$ for $r \geq 1$. From Lemma 3.2, we have

$$
E_{1}^{s+2, t(s), *}=\mathbb{Z}_{p}\{\mathbf{G} 1, \mathbf{G} 2, \cdots, \mathbf{G} 6\} .
$$

For the generator $\mathbf{G} 1$ with May filtration $M(\mathbf{G} 1)=9 s-18$ (see Lemma 3.3), we easily get that $\mathbf{G} 1$ is a permanent cycle in the MSS and converges to $\tilde{\delta}_{s} h_{1} h_{0}$, which equals $0 \in \operatorname{Ext}_{A}^{s+2, t(s)}\left(\mathbb{Z}_{p}, \mathbb{Z}_{p}\right)$ by $h_{1} h_{0}=0$. Then

$$
d_{r}(\mathbf{G} 1)=0
$$

and

$$
E_{r}^{s+2, t(s), 9 s-18} \subseteq \mathbf{Z}_{p}\{\mathbf{G} 1\}
$$

for $r \geq 1$. It follows that $h_{1,0} b_{1,0} a_{4}^{s-4} h_{4,0} h_{3,1} h_{2,2} h_{1,3} \notin d_{r}\left(E_{r}^{s+2, t(s), 9 s-18}\right)$ for $r \geq 1$.

For the generators $\mathbf{G} 2$ and $\mathbf{G} 3$ whose May filtrations are $M(\mathbf{G} 2)=M(\mathbf{G} 3)=$ $5 p+9 s-22$ (see Lemma 3.3), by the reason of May filtration, from (2.2) we see that $h_{1,0} b_{1,0} a_{4}^{s-4} h_{4,0} h_{3,1} h_{2,2} h_{1,3} \in E_{1}^{s+3, t(s), p+9 s-19}$, which represents that $h_{0} b_{0} \tilde{\delta}_{s} \in$ $\operatorname{Ext}_{A}^{s+3, t(s)}\left(\mathbb{Z}_{p}, \mathbb{Z}_{p}\right)$ in the MSS is not in $d_{1}\left(E_{1}^{s+2, t(s), 5 p+9 s-22}\right)$. Now we will show that

$$
E_{r}^{s+2, t(s), 5 p+9 s-22}=0
$$

for $r \geq 2$. By an easy calculation, from (2.3) and (2.4) one can have the first May differentials of $\mathbf{G} 2$ and $\mathbf{G} 3$ as follows:

$$
\begin{aligned}
d_{1}(\mathbf{G} 2)= & (-1)^{s+1} a_{4}^{s-4} b_{3,0} h_{3,1} h_{2,2} h_{1,3} h_{2,0} h_{1,0} \\
& +(-1)^{s} a_{4}^{s-4} b_{3,0} h_{4,0} h_{2,2} h_{1,3} h_{1,1} h_{1,0}+\cdots \\
\neq & 0, \\
d_{1}(\mathbf{G} 3)= & (-1)^{s+1} a_{4}^{s-4} b_{3,0} h_{3,1} h_{2,2} h_{1,3} h_{2,0} h_{1,0} \\
& +(-1)^{s+1} a_{4}^{s-4} b_{3,0} h_{4,0} h_{2,2} h_{1,3} h_{1,1} h_{1,0}+\cdots \\
\neq & 0 .
\end{aligned}
$$

It is easy to see that the first May differentials of $\mathbf{G} 2$ and $\mathbf{G} 3$ are linearly independent. Consequently, the cocycles of $E_{1}^{s+2, t(s), 5 p+9 s-22}$ must be zero. This means that

$$
E_{r}^{s+2, t(s), 5 p+9 s-22}=0
$$

for $r \geq 2$, from which we have that

$$
h_{1,0} b_{1,0} a_{4}^{s-4} h_{4,0} h_{3,1} h_{2,2} h_{1,3} \notin d_{r}\left(E_{r}^{s+2, t(s), 5 p+9 s-22}\right)
$$

for $r \geq 2$. In all, $h_{1,0} b_{1,0} a_{4}^{s-4} h_{4,0} h_{3,1} h_{2,2} h_{1,3} \notin d_{r}\left(E_{r}^{s+2, t(s), 5 p+9 s-22}\right)$ for $r \geq 1$.

For the generators $\mathbf{G} 6$ with May filtration $M(\mathbf{G} 6)=3 p+9 s-20$, by an easy calculation, from (2.3) and (2.4) we have the first May differentials of $\mathbf{G 6} 6$ as follows:

$$
d_{1}(\mathbf{G} 6)=(-1)^{s+1} a_{4}^{s-4} b_{2,1} h_{4,0} h_{3,1} h_{1,3} h_{1,1} h_{1,0}+\cdots \neq 0 .
$$


Thus

$$
E_{r}^{s+2, t(s), 3 p+9 s-20}=0
$$

for $r \geq 2$. At the same time, we also have that up to a nonzero scalar

$$
d_{1}(\mathbf{G} 6) \neq h_{1,0} b_{1,0} a_{4}^{s-4} h_{4,0} h_{3,1} h_{2,2} h_{1,3} \text {. }
$$

In summary, $h_{1,0} b_{1,0} a_{4}^{s-4} h_{4,0} h_{3,1} h_{2,2} h_{1,3} \notin d_{r}\left(E_{r}^{s+2, t(s), 3 p+9 s-20}\right)$ for $r \geq 1$.

Finally, we consider the generators $\mathbf{G} 4$ and $\mathbf{G} 5$ whose May filtrations are both $p+9 s-18$. By $(2.3)$ and (2.4), we have

$$
\begin{aligned}
& d_{1}(\mathbf{G} 4)=(-1)^{s+1} a_{4}^{s-4} b_{1,2} h_{4,0} h_{3,1} h_{2,2} h_{1,1} h_{1,0}+\cdots \neq 0 \\
& d_{1}(\mathbf{G} 5)=(-1)^{s+1} a_{4}^{s-4} b_{1,2} h_{4,0} h_{3,1} h_{1,3} h_{2,1} h_{1,0}+\cdots \neq 0 .
\end{aligned}
$$

The first May differential of each of the two generators contains at least a term which is not in the first May differential of the other generator. We can easily get that $d_{1}(\mathbf{G} 4), d_{1}(\mathbf{G} 5)$ and $h_{1,0} b_{1,0} a_{4}^{s-4} h_{4,0} h_{3,1} h_{2,2} h_{1,3}$ are linearly independent. Thus we have

$$
h_{1,0} b_{1,0} a_{4}^{s-4} h_{4,0} h_{3,1} h_{2,2} h_{1,3} \notin d_{1}\left(E_{1}^{s+2, t(s), p+9 s-18}\right) .
$$

Meanwhile, by (2.2), we also have that the permanent cycle

$$
h_{1,0} b_{1,0} a_{4}^{s-4} h_{4,0} h_{3,1} h_{2,2} h_{1,3} \notin d_{r}\left(E_{r}^{s+2, t(s), p+9 s-18}\right)
$$

for $r \geq 2$. In a word, $h_{1,0} b_{1,0} a_{4}^{s-4} h_{4,0} h_{3,1} h_{2,2} h_{1,3} \notin d_{r}\left(E_{r}^{s+2, t(s), p+9 s-18}\right)$ for $r \geq 1$.

From the above discussion, the permanent cycle $h_{1,0} b_{1,0} a_{4}^{s-4} h_{4,0} h_{3,1} h_{2,2} h_{1,3}$ cannot be hit by any differential in the MSS. Thus, $h_{1,0} b_{1,0} a_{4}^{s-4} h_{4,0} h_{3,1} h_{2,2} h_{1,3} \in$ $E_{1}^{s+3, t(s), p+9 s-19}$ converges nontrivially to $h_{0} b_{0} \tilde{\delta}_{s} \in \operatorname{Ext}_{A}^{s+3, t(s)}\left(\mathbb{Z}_{p}, \mathbb{Z}_{p}\right)$ in the MSS. Consequently, $h_{0} b_{0} \tilde{\delta}_{s} \neq 0$. This finishes the proof of Theorem 1.2.

\section{ACKNowledgement}

The authors would like to express their deep thanks to the referee, who read the manuscript of this paper and gave the authors many helpful comments and suggestions.

\section{REFERENCES}

[1] J. F. Adams, Stable homotopy and generalised homology, University of Chicago Press, Chicago, 1974. MR0402720(53:6534)

[2] T. Aikawa, 3-dimensional cohomology of the mod p Steenrod algebra, Math. Scand. 47 (1980), 91-115. MR600080 (82g:55023)

[3] X. Liu, A nontrivial product in the stable homotopy groups of spheres, Sci. China Ser. A 47 (2004), 831-841. MR2127211 (2005j:55016)

[4] X. Liu, On two non-trivial products in the stable homotopy groups of spheres, Bol. Soc. Mat. Mexicana(3) 13 (2007), 367-380.

[5] X. Liu, Some infinite elements in the Adams spectral sequence for the sphere spectrum, J. Math. Kyoto Univ. 48 (2008), 617-629.

[6] A. Liulevicius, The factorizations of cyclic reduced powers by secondary cohomology operations, Mem. Amer. Math. Soc. 42 (1962). MR0182001 (31:6226) 
[7] D. C. Ravenel, Complex cobordism and stable homotopy groups of spheres, Academic Press, Orlando, 1986. MR860042 (87j:55003)

[8] X. Wang, Q. Zheng, The convergence of $\tilde{\alpha}_{s}^{(n)} h_{0} h_{k}$, Sci. China Ser. A 41 (1998), 622-628. MR1633827 (99e:55022)

School of Mathematical Sciences and Laboratory of Pure Mathematics and Combinatorics, Nankai University, Tianjin 300071, People's Republic of China

E-mail address: xgliu@nankai.edu.cn, matlxg@hotmail.com

School of Mathematics, The University of Manchester, Oxford Road, Manchester, M13 9PL, United Kingdom

E-mail address: Hao.Zhao@manchester.ac.uk 